\title{
INVERSE KINEMATIC SOLUTION OF A 6 DOF SERIAL MANIPUlator
}

\author{
Andreea-Irina Afloare, Nicolae Apostolescu, \\ Alina-Ioana Chira \& Camelia-Elena Munteanu
}
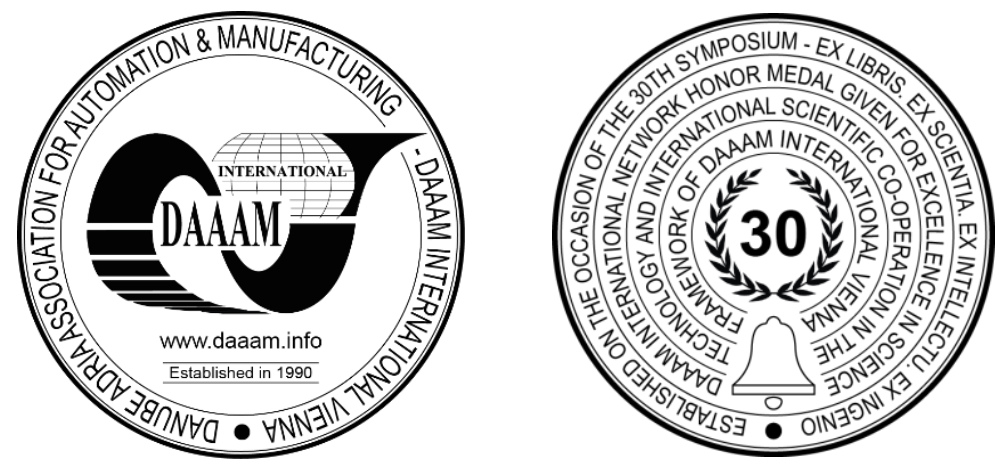

This Publication has to be referred as: Afloare, A[ndreea-Irina]; Apostolescu, N[icolae]; Chira, A[lina-Ioana] \& Munteanu, C[amelia] E[lena] (2019). Inverse Kinematic Solution of a 6 DoF Serial Manipulator, Proceedings of the 30th DAAAM International Symposium, pp.0628-0631, B. Katalinic (Ed.), Published by DAAAM International, ISBN 978 3-902734-22-8, ISSN 1726-9679, Vienna, Austria DOI: $10.2507 / 30$ th.daaam.proceedings.086

\begin{abstract}
In the robotics sector, inverse kinematics is one of the most typical research areas in design, trajectory planning, and dynamic analysis of robots. In this paper, an efficient algorithm for the inverse kinematics of a six degree of freedom robot manipulator for different aerospace applications is proposed. The proposed inverse kinematics algorithm is the Broyden-Fletcher-Goldfarb-Shanno algorithm, used for solving unconstrained nonlinear optimization problems. The developed algorithm is validated using simulation in Robotic toolbox. A separate MATLAB script is provided for a 3D visualization of the robot arm.
\end{abstract}

Keywords: robot manipulator; inverse kinematics; robotics; BFGS algorithm; end-effector; Robotics Toolbox.

\section{Introduction}

This paper deals with the initial study of a 6-DoF robotic flight simulator platform development taking into account a novel approach of the inverse kinematics process. Robot kinematics relate to the analytical study of the motion of a robot manipulator [1]. There are significantly two distinct spaces used in kinematics modeling of a serial robot, namely, Cartesian space and Quaternion space. The transformation between two Cartesian coordinate systems can be divided into a rotation and a translation. There are various ways to represent rotation; one of these representations is DenavitHartenberg [2], which shows that a global transformation between two joints requires four parameters that describe the robot kinematics.

The robot kinematics can be split into forward and inverse kinematics. The first one is straightforward, and there is no complexity in defining the equations. The complexity of the inverse kinematics problem of robot manipulators arises from their geometry and the inclusion of nonlinear equations. The difficulty of inverse kinematics is to find the joint variables that give the end-effector position and orientation. Inverse kinematics solutions for manipulators can be divided into analytical and numerical solutions [3].

The correlation between forward and inverse kinematics is presented in Fig. 1 [4]. 


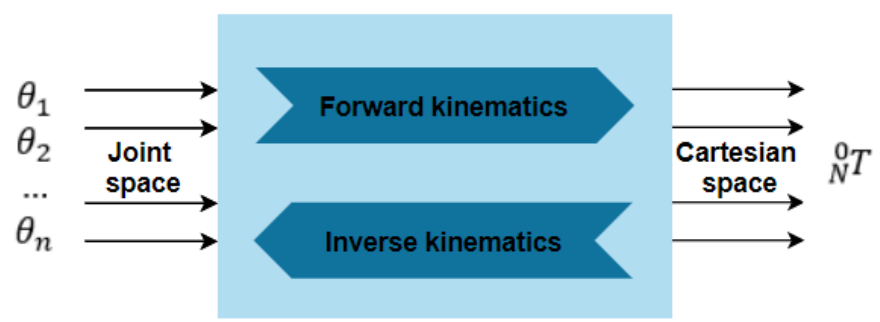

Fig. 1. Schematic description of forward and inverse kinematics

A significant role of various robotic manipulators is to reach the required position and orientation of the end-effector to complete a predefined task. The forward kinematic problem represents the set of relations that allow the definition of the position of the end-effector according to the articular coordinates, practically ensuring the conversion of the internal coordinates into external coordinates. Instead, the inverse kinematic problem provides the calculation of the joint coordinates that bring the end-effector to the desired position and orientation, giving the absolute coordinates.

In this paper, we will address the problem of position control and movement in joint space using a dedicated inverse kinematics algorithm. Trajectory tracking may consist of time parametrization throughout the path planning, but the reference trajectory in our study is defined in terms of the coordinates in robot workspace.

\section{Inverse kinematics algorithm}

The inverse kinematics solves problems like end-effector positions that are related to joint positions and can be reached in several configurations according to position vectors. Fig. 2 exposes the kinematic structure of the ABB IRB7600 -500 robot: Fig. 2a) shows the actuators of the robot placed at the joints between the links of the drive chain in series [5]; Fig. 2b) shows the Denavit-Hartenberg (D-H) mathematical representation type [6], and describe the spatial relationship between successive link coordinate frames.

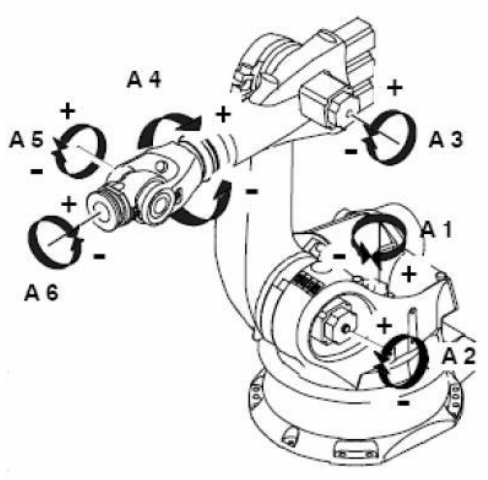

a)

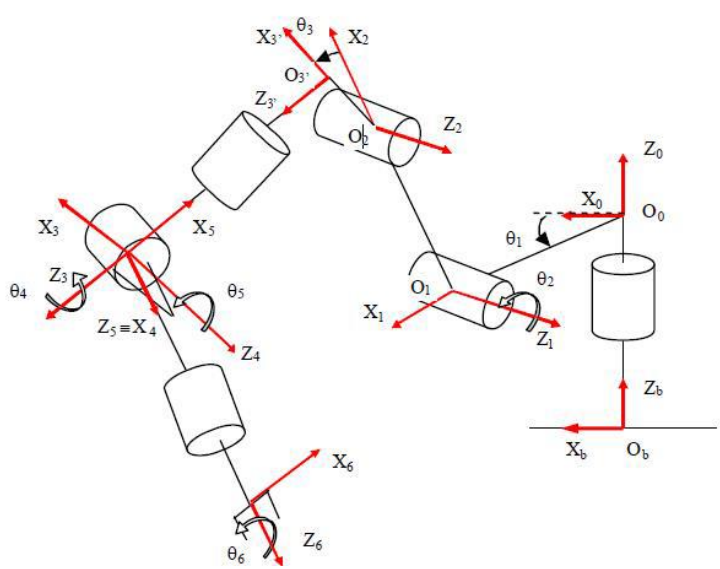

b)

Fig. 2. ABB IRB 7600 robot structure

To determine the values of the D-H parameters, some essential steps need to be followed to get the equation below:

$$
{ }_{i}^{i-1} T=T\left(d_{i}\right) T_{z}\left(\theta_{i}\right) T_{x}\left(\alpha_{i}\right) T_{x}\left(\alpha_{i}\right)=\left[\begin{array}{cccc}
\cos \theta_{i} & -\sin \theta_{i} & 0 & a_{i-1} \\
\sin \theta_{i} \cos \alpha_{i-1} & \cos \alpha_{i-1} \cos \theta_{i} & -\sin \alpha_{i-1} & -d_{i} \sin \alpha_{i-1} \\
\sin \theta_{i} \sin \alpha_{i-1} & \cos \theta_{i} \sin \alpha_{i-1} & \cos \alpha_{i-1} & d_{i} \cos \alpha_{i-1} \\
0 & 0 & 0 & 1
\end{array}\right]
$$

where ${ }_{i}^{i-1} T$ is a homogenous transformation matrix, $\alpha_{i-1}$ and $a_{i-1}$ are the translation and rotation about $x_{i-1}$ axis, respectively, $d_{i}$ and $\theta_{i-1}$ are the translation and rotation about $z_{i-1}$, respectively.

Then, the forward kinematics of the manipulator could be expressed by:

$$
{ }_{1}^{0} T\left(\theta_{1}\right){ }_{2}^{1} T\left(\theta_{2}\right){ }_{2}^{3} T\left(\theta_{3}\right){ }_{4}^{3} T\left(\theta_{4}\right){ }_{5}^{4} T\left(\theta_{5}\right){ }_{6}^{5} T\left(\theta_{6}\right)=T_{\text {end }}
$$

where $T_{\text {end }}$ is the configuration of the end-effector concerning the base frame. For inverse kinematics, $T_{\text {end }}$ is known; the focus is to calculate the joint angles $\theta_{i}$ through the matrix (2).

The D-H parameters of ABB 7600-500 serial robot are calculated as asserted in Table 1. After getting the parameters by using the D-H principle, the joint angle for a different position for inverse kinematics can be calculated. 


\begin{tabular}{|c|c|c|c|c|}
\hline Link & $\begin{array}{c}\text { Link length } \\
\boldsymbol{a}_{\boldsymbol{i}}[\mathbf{m}]\end{array}$ & $\begin{array}{c}\text { Twist link } \\
\boldsymbol{\alpha}_{\boldsymbol{i}}[\mathbf{d e g}]\end{array}$ & $\begin{array}{c}\text { Link offset } \\
\boldsymbol{d}_{\boldsymbol{i}}[\mathbf{m}]\end{array}$ & $\begin{array}{c}\text { Joint angle } \\
\boldsymbol{\theta}_{\boldsymbol{i}}[\mathbf{d e g}]\end{array}$ \\
\hline 1 & 0.41 & -90 & 0.78 & 0 \\
\hline 2 & 1.075 & 0 & 0 & -90 \\
\hline 3 & 0.165 & -90 & 0 & 0 \\
\hline 4 & 0 & 90 & 1.056 & 0 \\
\hline 5 & 0 & -90 & 0 & 0 \\
\hline 6 & 0 & 0 & 0.25 & 180 \\
\hline
\end{tabular}

Table 1. DH parameters of the IRB $7600-500$ serial robot

\section{Simulation and results}

The main aim of this study is to solve 6 DoF serial robots inverse kinematics problem using the BFGS algorithm and to simulate the solution of the inverse kinematics by using two different simulation environments: MATLAB and Robot Studio.

The first simulation process utilizes Matlab Robotics System Toolbox to verify the position of the end-effector and to determine the joint angles using the algorithm above for each point set in the proposed trajectory. The verification of the attitude control involved the analysis of the homogeneous transformation matrix.

The second simulation process uses the RAPID program in Robot Studio, to validate the results recorded in the first simulation, using the same algorithm. Verification of the inverse kinematics method using the Robot Studio software was done for the ABB7600-500 robot and the controller associated with it.

The trajectory and motion of the robot, as well as the tracking of the joint angle, are described in the RAPID programming language that writes the data for analysis. The trajectory can be defined punctually, and when moving from one point to another, the joint angles are determined according to the position of the axis. The determination of the end-effector's position and attitude was performed with special functions from the Robot Studio program (CalcRobT and CalcJointT). Robot Studio functions find the trajectory defined through different five points, and the end-effector position for these points are shown in Table 2. The last three columns in the below table highlight the deviations between trajectory points and end-effector position.

\begin{tabular}{|c|c|c|c|c|c|c|c|c|c|}
\hline \multirow{2}{*}{ Point } & \multicolumn{3}{|c|}{ Trajectory points $[\mathbf{m m}]$} & \multicolumn{3}{|c|}{ End-effector position $[\mathbf{m m}]$} & \multicolumn{3}{c|}{ Deviation [mm] } \\
\cline { 2 - 10 } & $\mathbf{X}$ & $\mathbf{y}$ & $\mathbf{z}$ & $\mathbf{x}$ & $\mathbf{y}$ & $\mathbf{z}$ & $\boldsymbol{\Delta x}$ & $\boldsymbol{\Delta y}$ & $\boldsymbol{\Delta} \mathbf{z}$ \\
\hline $\mathbf{1}$ & 1900.0 & 100.0 & 1133.3 & 1899.85 & 100.227 & 1133.52 & 0.1500 & -0.2270 & -0.2200 \\
\hline $\mathbf{2}$ & 1838.4 & 332.4 & 1128.3 & 1838.60 & 331.704 & 1128.32 & -0.2000 & 0.6960 & -0.0200 \\
\hline $\mathbf{3}$ & 1677.8 & 502.8 & 1113.2 & 1678.12 & 502.442 & 1113.18 & -0.3200 & 0.3580 & -0.0200 \\
\hline $\mathbf{4}$ & 1469.9 & 585.0 & 1088.7 & 1470.18 & 584.900 & 1088.67 & -0.2800 & 0.1000 & 0.0300 \\
\hline $\mathbf{5}$ & 1258.9 & 587.8 & 1055.3 & 1258.90 & 587.800 & 1055.30 & 0 & 0 & 0 \\
\hline
\end{tabular}

Table 2. ABB IRB 7600 - End-effector position for a specified trajectory

For each of the 1, 3 and 5 points from the trajectory, the values of the joint angles (Joint Angles C) were calculated through inverse kinematics and compared with those obtained by simulating the movement of ABB IRB7600 serial robot using Robot Studio driven by a RAPID program (Joint Angles R).

\begin{tabular}{|c|c|c|c|c|c|c|c|c|}
\hline \multicolumn{3}{|c|}{ Point 1 } & \multicolumn{3}{c|}{ Point 3 } & \multicolumn{3}{c|}{ Point 5 } \\
\hline $\begin{array}{c}\text { Joint } \\
\text { Angle C }\end{array}$ & $\begin{array}{c}\text { Joint } \\
\text { Angle R }\end{array}$ & $\begin{array}{c}\Delta \text { J Joint } \\
\text { Angle }\end{array}$ & $\begin{array}{c}\text { Joint Angle } \\
\text { C }\end{array}$ & $\begin{array}{c}\text { Joint } \\
\text { Angle R }\end{array}$ & $\begin{array}{c}\boldsymbol{\Delta}_{-} \text {Joint } \\
\text { Angle }\end{array}$ & $\begin{array}{c}\text { Joint } \\
\text { Angle C }\end{array}$ & $\begin{array}{c}\text { Joint } \\
\text { Angle R R_ }\end{array}$ & $\begin{array}{c}\boldsymbol{\Delta}_{-} \text {Joint } \\
\text { Angle }\end{array}$ \\
\hline 3.4759 & 3.4682 & 0.0077 & 19.3838 & 19.3997 & -0.0159 & 30.2200 & 30.2257 & -0.0057 \\
21.4093 & 21.4218 & -0.0125 & 6.0040 & 15.9947 & 0.0093 & 2.5363 & 2.5247 & 0.0116 \\
24.7475 & 24.7419 & 0.0056 & 33.8021 & 33.8135 & -0.0114 & 54.6952 & 54.7075 & -0.0123 \\
4.8087 & 4.8028 & 0.0060 & 24.7384 & 24.7495 & -0.0111 & 34.7130 & 34.7169 & -0.0039 \\
-46.2650 & -46.2643 & -0.0007 & -52.4983 & -52.5038 & 0.0055 & -62.1140 & -62.1179 & 0.0039 \\
-3.3290 & -3.3244 & -0.0046 & -15.6684 & -15.6748 & 0.0064 & -17.9533 & -17.9535 & 0.0002 \\
& & & & & & & & \\
\hline
\end{tabular}

Table 3. Values of the manipulator's joint angles

The graphical results of the predefined trajectory into Matlab and Robot Studio environments for inverse kinematics simulations are exposed in Fig. 3. 


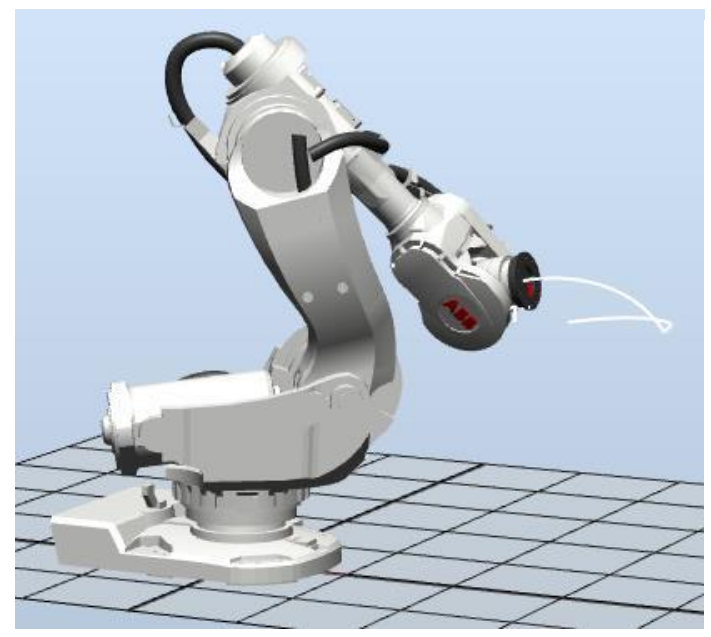

a) Robot Studio

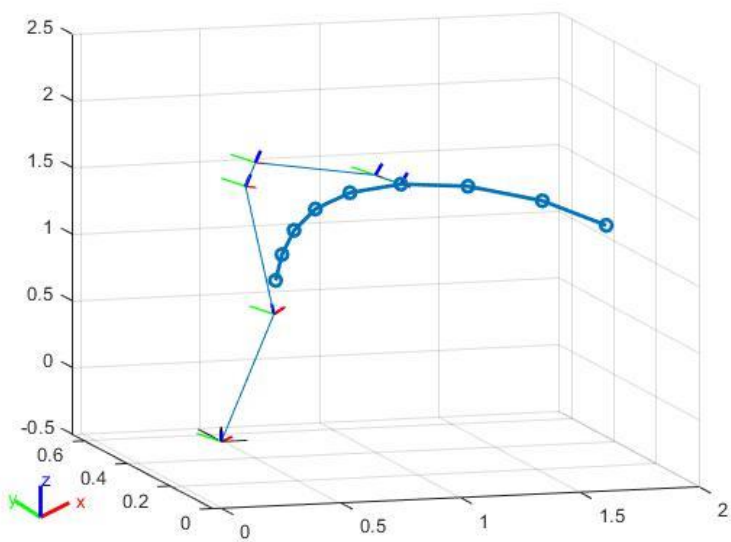

b) Matlab

Fig. 3. ABB IRB 7600-500 robot trajectory

\section{Conclusion}

To conclude, this paper proposed an analytical solution of inverse kinematics algorithm for the ABB IRB7600-500 serial manipulator, using an iterative method for solving unconstrained nonlinear optimization problems.

A geometric approach of various links of the manipulator was considered to obtain the solution. The inverse kinematics model was simulated in two different environments: MATLAB Robotics toolbox and Robot Studio.

The results showed that the end-effector moved along a predefined trajectory, confirming the efficacy of the model. Also, it was developed a 3D graphical representation of the joint position with respect to time, to verify the deviation. The accuracy of the model used in simulations confirmed the theoretical assumptions under a given trajectory. In the future, this work will be integrated in the robotic flight simulator model to improve the quality of the simulation environment. One objective is to develop an appropriate pilot model to analyse the human performance envelope.

\section{Acknowledgments}

The work has been funded by the Romanian Government through the Ministry of Research and Innovation (MCI), under the "AEROEXPERT 2019-2022" program, financial agreement no. 8N/2019.

\section{References}

[1] Tourissis, V. D. \& Ang, M. H. (1989). A Modular Architecture for Inverse Robot Kinematics, IEEE Transaction on Robotics and Automation

[2] Denavit, J. \& Hartenberg, R. S. (1955). A kinematic notation for lower-pair mechanisms based on matrices, Journal of Applied Mechanics

[3] Tolani, D., Goswami, A. \& Badler, N. I. (2000). Real-time inverse kinematics techniques for anthropomorphic limbs, Graphical Models, https://doi.org/10.1006/gmod.2000.0528

[4] Chen, Q., Zhu, S. \& Zhang, X. (2015). Improved inverse kinematics algorithm using screw theory for a six-DOF robot manipulator, Int. J. Adv. Robot. Syst

[5] https://new.abb.com/

[6] Schilling, R. J. (1990). Fundamentals of Robotics, Prentice Hall, USA 